\title{
Torsional MEMS scanner design for high-resolution display systems
}

\author{
Hakan Urey \\ Microvision Inc., 19910 North Creek Pkwy, Bothell, WA 98011, USA \\ Currently with: KOÇ University, Sariyer, Istanbul, TURKEY
}

\begin{abstract}
In scanning display systems, high horizontal and vertical resolution, and high refresh rate requirements translate into large mirror-size scan-angle product and high scanner-frequency requirements. A comparison between published scan-angle mirror-size product values for MEMS scanners and a Steel mechanical scanner is presented. Current performance levels of Steel mechanical scanners are better; however, Silicon MEMS scanners have good material properties and should be able to reach and exceed the performance levels of conventional mechanical scanners. The resolution limitations of mechanical and MEMS scanners are established using dynamic mirror deformation, flexure stress, and other oscillation mode frequencies. Analytical formulas for torsional, vertical deflection mode, lateral deflection mode, and rocking mode natural frequencies are derived using mechanical beam deflection theory.
\end{abstract}

Keywords: Resonant scanners, MOEMS, RSD, eigenmodes, torsion, natural frequency

\section{INTRODUCTION}

Retinal Scanning Displays (RSD) operate by raster-scanning a modulated (either directly or externally) point light source onto the viewer's retina. RSD operation and system architecture is discussed in detail elsewhere ${ }^{1,2,3}$. RSDs typically employ two uniaxial or one biaxial scanner where the horizontal scanner is operated at resonance, and the vertical scanner is driven by a sawtooth waveform ${ }^{4,5}$. The combined motions of the two scanners create a 2-D sinusoidal raster.

There are several laser scanning display implementations using non-MEMS scanners, such as acoustooptic scanners ${ }^{6}$, polygonal scanners ${ }^{7}$, and galvanometric and resonant scanners ${ }^{8,9}$. MEMS scanners were mostly developed during the past two decades. MEMS technology can be used to fabricate the scanner, actuator, and scanner position sensors all integrated on the same chip in one package, which then becomes the heart of the scanning display system. Most of the MEMS scanners are low-inertia galvanometric or resonant type scanners.

The line-rate of an SVGA (800x600 pixels), $60 \mathrm{~Hz}$ refresh rate display is 36,000 lines per second. Using a bidirectional-scanning architecture, a horizontal scanner operating at about 19 to $20 \mathrm{KHz}$ is required (18,000 cycles for display plus some time for scanner retraces). At such high frequencies, the only way to lower the power requirement is to take advantage of the mechanical gain, or the Q-factor, of the scanner by operating the scanner at its mechanical resonant frequency. Resonant sinusoidal motion of the scanner takes up the least amount of power but results in pixel size and brightness variations across the scan line due to scanner speed variations. Typically, writing is halted during the extremities of the scan line to minimize the speed variation. The pixel size and brightness variations during the visible portion of the scan can be easily corrected electronically.

A resonant high-frequency horizontal scanner and a linear-ramp driven vertical scanner are the desired drive waveforms for current RSD systems. This configuration takes advantage of low power operation for the horizontal scanner and allows for good control of the vertical scanner relative to the horizontal 
scanner without requiring too much power, as it operates at a much lower frequency compared to the horizontal scanner.

As discussed in Section 2, scan-angle mirror-size product $(\theta D)$ determines the resolution of scanning systems. In section 3, $\theta D$ metric is used to compare MEMS scanners in the literature. Steel based nonMEMS scanner, which has superior performance compared to MEMS scanners, is also included in the comparison. In the remainder of the paper, we discuss mechanical design tradeoffs such as mode frequencies, dynamic deformation, and stress, and show that for those mechanical trades, Silicon has better properties than Steel. We then conclude that Silicon MEMS scanners have the potential to provide very high resolution and high frequency scanners for scanning display systems. Scanner oscillation mode frequencies are discussed in detail and approximate analytical formulas that can be used to make first order approximations are derived. Note that, drive voltage and power requirements, which are dependent on the actuator type, mirror properties, and damping are not included in this discussion.

\section{SCANNER RESOLUTION}

For display systems, the point spread function (PSF) and display modulation transfer function (MTF) are determined by diffraction from the aperture stop in the system and by system aberrations. If aberrations are controlled, then the system will be limited only by diffraction. To accommodate various aperture shapes and illumination conditions, an aperture shape factor $a$ may be introduced to relate beam spot size $s$ to the optical wavelength $\lambda$ and system f-number $f_{\#}$ according to ${ }^{10}$

$$
s=a \lambda f_{\#}
$$

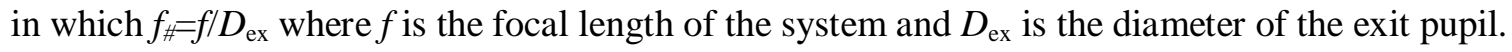

Various definitions for spot size may be used, including full width at half maximum power, the $1 / e^{2}$ intensity diameter, or $M T F=0.5$ (which specifies the spatial period of a sinusoidal grating resolved by the system with $50 \%$ contrast ratio). The most useful definition will depend on the system function, and $a$ values can range from 0.75 to 2 or more depending on the definition of spot size, the aperture shape, and Gaussian beam clipping at the system apertures.

If the scanner serves as the aperture stop of the system, then the number of resolvable spots can be rewritten in terms of the optical full-range scan angle $\theta_{\text {opt }}$, the mechanical zero-to-peak angle $\theta_{\text {mech }}$, and the mirror diameter $D$ according to

$$
N=\frac{\theta_{\mathrm{opt}} D}{a \lambda}=\frac{4 \theta_{m e c h} D}{a \lambda}
$$

There are additional factors such as mirror overscan factor and incoming beam angle that need to be taken into account in calculating the scanner resolution for displays applications. A more detailed resolution equation is presented in Ref. 4. The number of resolvable spots for a coherent focusing geometry system that is diffraction limited thus depends on the $\theta D$-product of the scanner. Note that, for center-pivot scanners, $\theta D$ also corresponds to the motion of the edge of the scanner. 
Table 1: $\theta D$-product and scanner frequency requirements for various display formats (adapted from Ref. 4)

\begin{tabular}{|l|r|r|r|r|r|r|r|}
\hline Display Format & QVGA & VGA & SVGA & XGA & SXGA & UXGA & HDTV \\
\hline$N_{h}$ - Horizontal resolution & 320 & 640 & 800 & 1024 & 1280 & 1600 & 1920 \\
\hline$N_{V}$ - Vertical resolution & 240 & 480 & 600 & 768 & 1024 & 1200 & 1080 \\
\hline$\theta_{M M S A} \cdot D$ (deg.mm) & 3.9 & 7.8 & 9.7 & 12.4 & 15.6 & 19.4 & 23.3 \\
\hline$f_{\mathrm{s}}$ - Horizontal scanner freq. $(\mathrm{Hz})$ & 8000 & 16000 & 20000 & 25600 & 34133 & 40000 & 36000 \\
\hline
\end{tabular}

\section{SCANNER COMPARISON}

Figure 1 shows a graph of requirements and measured scanner $\theta D$-product and horizontal resonant scanner frequency from a number of research publications ${ }^{4,11-22}$. Published scan angles are all converted to 0 -to-peak mechanical scan angle. The list is not a complete list of video scanner references. There are some references that cannot be included in the figure due to the lack of clarity about the scan angle definition, and there may be some others not known to the author. To meet a given video standard, a scanning display must fall above and to the right of the indicated lines. Scanner has to meet the frequency requirement but somewhat smaller $\theta \mathrm{D}$ might be acceptable at the expense of display MTF or resolvability of high-spatial frequency features.

The scanner with the highest $\theta D$-product is a non-MEMS mechanical resonant scanner (MRS), illustrated in Figure 2(a) (labeled Urey 2002 in the figure below ${ }^{11}$ ). The MRS is made of Steel and has good magnetic and mechanical properties. The MRS can achieve SXGA resolution by scanning two beams in parallel, which reduces the SXGA frequency requirement to 16KHz. The MRS has been used in all RSDs used in military helmet-mounted display (HMD) systems. As seen from the figure, MRS has significantly better performance compared to the best performing Silicon MEMS scanners, which is the Microvision MEMS scanner used in SVGA resolution NOMAD display product ${ }^{23}$. The performance difference is not due to inferior material properties for Silicon, but rather due to sub-optimal designs, and fabrication and packaging problems.

Figure 2(a) and (b) illustrate Microvision MRS and biaxial MEMS scanner. MRS is actuated using two electromagnets and operates in resonant torsional mode. Permanent magnets, U-core, scan mirror, and the base plate are all part of the magnetic circuit. The scan mirror has high magnetic permeability and the magnets at the end points of the torsion arm create a magnetic flux through the gaps on both ends between the mirror plate and the U-core. The constant DC flux creates a downward force on either end of the mirror plate and pulls the mirror down; but the net torque or twisting moment is zero. When opposing currents flow through the coils, the force is no longer balanced and the mirror starts to tilt in response to the current. When an AC current is applied, the mirror pivots periodically and can act as a scanner.

The biaxial MEMS scanner horizontal axis is actuated electrostatically using a parallel plate actuator and the vertical axis is actuated magnetically using Lorentz force. The mirror is etched on Silicon using deep reactive ion etching (DRIE) to tightly control the flexure and mirror dimensions. Vertical drive coils can be gold, copper, or some of the other metal. There are two permanent magnets on either side of the vertical scan frame (not shown in the figure) to produce a static magnetic field in the gap between the magnets. The cross product of the magnetic field along the horizontal axis and the current along the vertical axis produces a force perpendicular to the page. The opposite current direction on different sides of the coil generates forces into and out of the page, resulting in a net torque or twisting moment on the torsional scan mirror. 
MRS has better resolution and lower cost in small quantities compared to MEMS scanners. MEMS technology offers low-cost high-volume fabrication capability and allows biaxial scanner implementation, which reduces the size of the display system. On the other hand, due to long fabrication lead times, and packaging and yield problems, there are only very few successful optical MEMS products in the market (e.g. Texas Instruments DMD technology based DLPTM projection display systems). Microvision NOMAD display system, which was launched recently, is another example of MEMS-based display system.



Figure 1: Scanner comparison. All are MEMS scanners except Urey (2002), which is made of Steel. 


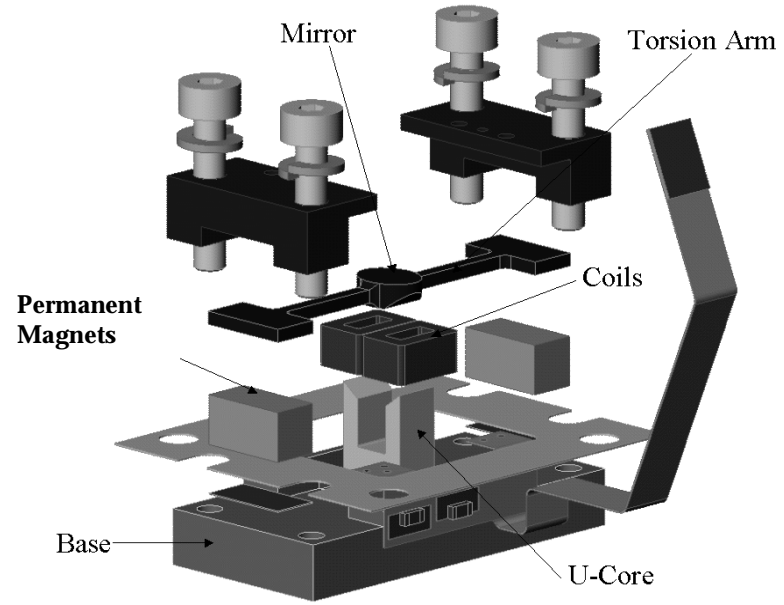

(a)

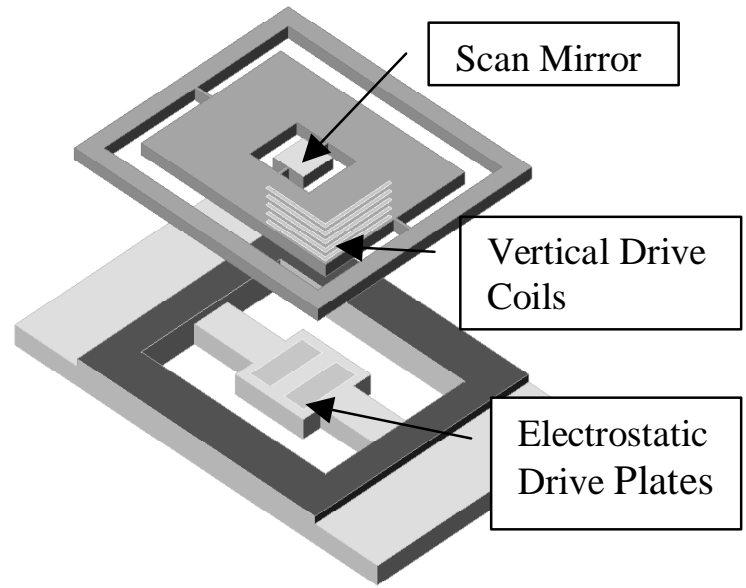

(b)

Figure 2 Microvision scanners: (a) Uniaxial Mechanical Resonant Scanner (MRS), (b) Biaxial MEMS scanner

\section{OSCILLATION MODE NATURAL FREQUENCIES}

Figure 3 shows a rectangular-block torsional scan-mirror and Figure 4 illustrates the four oscillation modes that we refer to as the torsional, vertical, horizontal, and rocking modes. The torsional and other oscillation mode natural frequencies can be calculated using the scanner dimensions and material properties. The natural frequency for an oscillation mode $(\omega)$ can be calculated by obtaining a second order differential equation in the form:

$$
y^{\prime \prime}+\omega^{2} y=0
$$

Table 2 summarizes the spring constant, mirror inertia, and natural frequency formulas for the torsional scanner. We assume that the entire bending take place at the flexures (i.e. mirror is rigid) and the mass of the flexures is negligible. This approximation is not generally valid especially when the flexure length is not very long and flexure width is not very small compared to the scan mirror length. The formulas can be used to predict the order of different modes and the relative distance between modes. Once a mirror size design point is reached, FEA should be used to verify the design.

Note that these mode frequency formulas are for rectangular block mirror and rectangular cross-section flexures. Other flexure and mirror shapes and shifts in axis of rotation can also be analyzed by substituting appropriate values of $M, I_{m}, I_{s}$, and $I_{p}$ for the mirror and the flexures in the corresponding formulas.

Torsional Mode: Torsional spring constant is proportional to the polar moment of inertia $\left(I_{p}\right)$ of the flexure cross-section and torsional modulus of the material $(G)$ and inversely proportional to the flexure length $\left(L_{f}\right)$. The shape factor for square cross-section beam is found in Ref. 24 (formula is given for $a>\mathrm{b}$, if $b>a$, then a and $\mathrm{b}$ values should be interchanged in $I_{p}$ and $K_{a b}$ formulas). If a net torque $T$ is applied to the mirror around the $x$-axis, then the following relationship can be used to compute the deflection angle $\theta$ and the natural frequency $\omega$ of the torsional mode:

$$
T=\frac{2 G I_{p} \theta}{L_{f}}=-I_{m} \theta^{\prime \prime}
$$




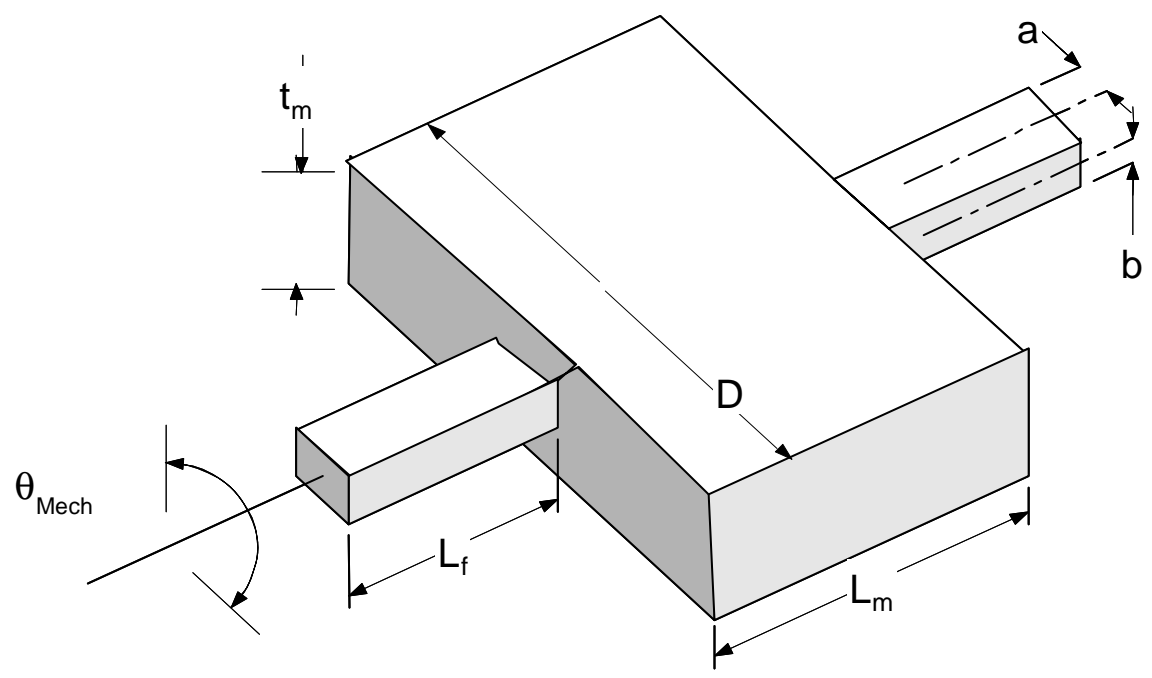

Figure 3: Torsional resonant scanner geometry


Figure 4 Four different oscillation modes for torsional scanner 
Vertical Mode: Assume a force $F$ in the vertical direction (along the z-axis) is applied to the mirror. The vertical deflection along the flexure $z(x)$ and tip deflection can be found using the following differential equation and the boundary conditions:

$$
\begin{aligned}
& z^{\prime \prime \prime}=\frac{-F}{2 E I_{s}} \\
& z(0)=0 ; \quad z^{\prime}(0)=0 ; \quad z^{\prime}\left(L_{f}\right)=0 \\
& z(x)=\frac{F}{2 E I_{s}}\left(\frac{x^{3}}{6}-\frac{L_{f} x^{2}}{4}\right) \\
& z_{\max }=z(L)=\frac{F L_{f}^{3}}{E I_{s}}
\end{aligned}
$$

where $E$ is the Young's modulus and $I_{s}$ is the moment of inertia for the flexure cross-sectional area. $E I$ product is the flexural rigidity of the beam and is a measure of the resistance of a beam to bending.

Horizontal Mode: Horizontal mode of oscillation moves the mirror in $y$-axis. The corresponding mode frequency formulas are similar to that of the vertical mode. The only difference is the flexure width $a$ and flexure thickness $b$ are interchanged. Thus for square cross-section flexures, the horizontal and vertical mode frequencies should be identical.

Rocking Mode: Assume a torque $T$ around $y$-axis (perpendicular to the torsional scan axis) is applied to the mirror:

$$
T=I_{m} \theta^{\prime \prime}
$$

where $I_{m}$ is the mirror mass moment of inertia for rotation around y-axis and $\theta$ is the rotation angle of the mirror in rocking mode. The beam deflection $y(x)$ can be found using the following differential equation and the boundary conditions:

$$
\begin{gathered}
y^{\prime \prime}\left(L_{f}\right)=\frac{\mathrm{T}}{E I_{s}}=\frac{I_{m} \theta^{\prime \prime}}{E I} \\
y(0)=0 ; \quad y^{\prime}(0)=0 ; \\
y\left(L_{f}\right)=L_{m} \theta / 2 ; \quad y^{\prime}\left(L_{f}\right)=-\theta \\
y(x)=\frac{-\theta\left(L_{f}+L_{m}\right)}{L_{f}^{3}} x^{3}+\frac{\theta\left(L_{f}+1.5 L_{m}\right)}{L_{f}^{2}} x^{2} \\
y^{\prime \prime}(L)=\frac{-\theta}{L^{2}}\left(4 L_{f}+3 L_{m}\right)
\end{gathered}
$$

Note that similar formulas can also be derived for the horizontal rocking mode (not shown in Figure 4). Horizontal rocking mode frequency is typically higher than the vertical rocking frequency and the results are not included here. 
Table 2: Natural frequencies for different oscillation modes for torsional scanners $(2 a=$ width; $2 b=$ thickness of flexural beam)

\begin{tabular}{|c|c|c|c|}
\hline & $\begin{array}{l}\text { Mirror (assume } \\
\text { rectangular } \\
\text { block) }\end{array}$ & $\begin{array}{l}\text { Spring Constant, } K_{s} \\
\text { Flexure spring Inertia, } I_{s} \\
\text { Flexure polar moment of inertia, } I_{p} \\
\text { (for rectangular cross-section) }\end{array}$ & $\begin{array}{l}\text { Resonant Frequency } \omega \text {, } \\
\qquad[\omega=2 \pi f]\end{array}$ \\
\hline $\begin{array}{l}\text { Torsional } \\
\text { mode }\end{array}$ & $\begin{array}{l}\text { Mirror Inertia } \\
I_{m}=\frac{1}{12} \rho t L_{m} D^{3}\end{array}$ & $\begin{array}{c}\text { For } a>b ; K_{s}=\frac{2 I_{p} G}{L_{f}} \\
I_{p}=K_{a b} a b^{3}, \text { where } \\
K_{a b}=\left(5.33-3.36 \frac{b}{a}\left(1-\frac{b^{4}}{12 a^{4}}\right)\right)\end{array}$ & $\sqrt{\frac{K_{s}}{I_{m}}}=\sqrt{\frac{24 K_{a b} G a b^{3}}{L_{f} \rho t L_{m} D^{3}}}$ \\
\hline $\begin{array}{l}\text { Vertical } \\
\text { mode }\end{array}$ & $\begin{array}{l}\text { Mirror mass } \\
M=\rho t L_{m} D\end{array}$ & $\begin{array}{c}K_{s}=\frac{24 E I_{s}}{L_{f}^{3}} \\
I_{s}=\frac{(2 a)(2 b)^{3}}{12}=\frac{4 a b^{3}}{3}\end{array}$ & $\sqrt{\frac{K_{s}}{M}}=\sqrt{\frac{32 E a b^{3}}{L_{f}^{3} \rho t L_{m} D}}$ \\
\hline $\begin{array}{l}\text { Horizont } \\
\text { al mode }\end{array}$ & $\begin{array}{l}\text { Mirror Mass } \\
M=\rho t L_{m} D\end{array}$ & $\begin{aligned} K_{s} & =\frac{24 E I_{s}}{L_{f}^{3}} \\
I_{s} & =\frac{4 a^{3} b}{3}\end{aligned}$ & $\sqrt{\frac{K_{s}}{M}}=\sqrt{\frac{32 E a^{3} b}{L_{f}^{3} \rho t L_{m} D}}$ \\
\hline $\begin{array}{l}\text { Rocking } \\
\text { mode }\end{array}$ & $\begin{array}{l}\text { Mirror Inertia } \\
I_{m}=\frac{1}{12} \rho t L_{m}^{3} D\end{array}$ & $\begin{array}{c}K_{s}=\frac{E I_{s}\left(4 L_{f}+3 L_{m}\right)}{L_{f}^{2}} \\
I_{s}=\frac{4 a b^{3}}{3}\end{array}$ & $\sqrt{\frac{K_{s}}{I_{m}}}=\sqrt{\frac{16 E\left(4 L_{f}+3 L_{m}\right) a b^{3}}{\rho t D L_{m}^{3} L_{f}^{2}}}$ \\
\hline
\end{tabular}

Table 3 Ratio of different mode frequencies to torsional mode frequency for Silicon and Steel. Assumed rectangular cross-section for flexures and rectangular block mirror.

\begin{tabular}{|c|c|c|c|}
\hline & $\omega_{\text {mode }} / \omega_{\text {orsion }}$ & $\begin{array}{c}\text { Frequency ratio for } \mathrm{Si} \\
E=170 \mathrm{GPa} \\
G=51 \mathrm{GPa}\end{array}$ & $\begin{array}{c}\text { Frequency ratio for } \\
\text { Steel } E=203 \mathrm{GPa} \\
G=78.5 \mathrm{GPa}\end{array}$ \\
\hline Horizontal Mode & $\sqrt{\frac{4 E}{3 K_{a b} G}} \frac{D}{L_{f}}$ & $\begin{array}{l}1.4 \frac{D}{L_{f}} \quad \text { if } a=b \\
1.1 \frac{D}{L_{f}} \quad \text { if } a=2 b\end{array}$ & $\begin{array}{l}1.24 \frac{D}{L_{f}} \quad \text { if } a=b \\
0.97 \frac{D}{L_{f}} \quad \text { if } a=2 b\end{array}$ \\
\hline Vertical Mode & $\sqrt{\frac{4 E}{3 K_{a b} G}} \frac{D a}{L_{f} b}$ & $\begin{array}{l}1.4 \frac{D}{L_{f}} \quad \text { if } a=b \\
2.2 \frac{D}{L_{f}} \quad \text { if } a=2 b\end{array}$ & $\begin{array}{l}1.24 \frac{D}{L_{f}} \quad \text { if } a=b \\
1.94 \frac{D}{L_{f}} \quad \text { if } a=2 b\end{array}$ \\
\hline Rocking Mode & $\sqrt{\frac{8 E\left(1+\frac{3 L_{m}}{4 L_{f}}\right)}{3 K_{a b} G}} \frac{D}{L_{m}}$ & 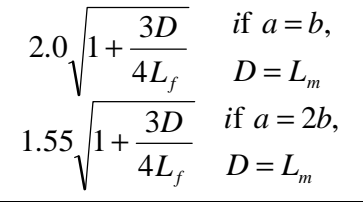 & $\begin{array}{l}1.75 \sqrt{1+\frac{3 D}{4 L_{f}} \quad} \quad \begin{array}{l}D=L_{m} \\
1.37 \sqrt{1+\frac{3 D}{4 L_{f}}} \quad \begin{array}{l}\text { if } a=2 b, \\
D=L_{m}\end{array}\end{array}\end{array}$ \\
\hline
\end{tabular}


Mode frequencies should be well separated from the torsional mode frequency and its harmonics in order to minimize power dissipation. Horizontal and vertical modes do not change the scanned beam direction. Rocking-mode, however, deflects the incoming beam perpendicular to the intended scan axis, creating an undesired off-axis motion that degrades the display image quality. Biaxial scanners should be designed such that cross coupling between the torsional and rocking modes of inner and outer scan frames are avoided.

Mirror parameters should be chosen such that rocking, horizontal, and vertical modes are not close to the torsional mode frequency or its harmonics. Table 3 presents simplified formulas for calculating the ratio of the undesired mode frequencies to the torsional mode frequency with $D / L_{f}$ as the parameter. Mode frequency ratio for Silicon is $13 \%$ larger than that of Steel for all modes. To obtain high Q-factor, it is desired to have the torsional mode as the first mode (mode with the smallest frequency) and to have the mode frequency ratios as large as possible. In some MRS designs, torsional mode is not the first mode but still modest Q-factor values can be obtained.

\section{OTHER DESIGN CONSIDERATIONS}

Dynamic Deformation:

Both static flatness and dynamic flatness of the scanning mirror are important for image quality. Unlike surface micromachined mirrors, surface flatness for bulk micromachined mirrors can be very good (as good as the surface flatness of the original substrate). High acceleration forces during mechanical deflection of the scan mirror result in bending of the mirror. Even very small amount of deviation from linearity due to mechanical deformation can result in optical distortion of the pixel and the image. Maximum deformation occurs at the extremities of the scan. For rectangular block mirrors, the following formulas can be used to compute the maximum mirror deformation $\delta_{\max }$, which is defined as the deviation from linearity ${ }^{25}$

$$
\delta_{\max }=0.217 \frac{\rho f^{2} D^{5} \theta_{\text {mech }}}{E t^{2}}
$$

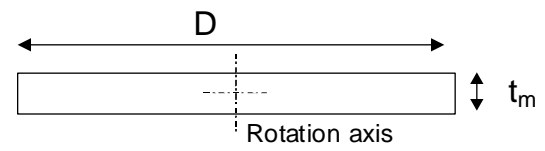

$f:$ the scanner frequency

$\theta_{\text {mech }}$ : peak mechanical scan angle

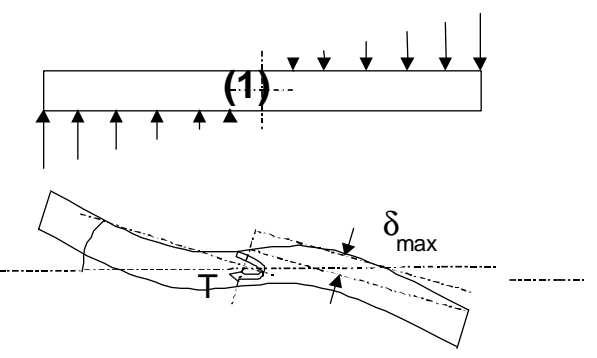

Stress on the flexures:

Another consideration is the stress on the flexures. The maximum stress should be smaller than the yield stress $\left(S_{y}\right)$ of the material. The maximum stress in a rectangular cross-section torsional flexure is given by: ${ }^{24}$

$$
\tau_{\text {MAX }}=\frac{3 K_{a b} G b \theta_{\text {mech }}}{8 L_{f}} \cdot\left[1+0.6095\left(\frac{b}{a}\right)+0.8865\left(\frac{b}{a}\right)^{2}-1.8023\left(\frac{b}{a}\right)^{3}+0.91\left(\frac{b}{a}\right)^{4}\right] \quad \text { for } a \geq b
$$




\section{MATERIAL COMPARISON}

Table below compares different material property related coefficients for single crystal Silicon and a kind of Carbon Steel. If a bulk-micromachined torsional Silicon scanner and a conventionally machined torsional Steel scanner with the same dimensions are produced, Silicon scanner would have $47 \%$ higher resonant frequency, 67\% higher frequency for other modes (thus better mode separation if torsional mode is the $1^{\text {st }}$ mode), about $1 / 3$ of the dynamic deformation of the Steel mirror, and $32 \%$ higher angle of rotation before flexures break.

\section{Table 4 Material property coefficients for Silicon and Steel torsional scanner performance} parameters

\begin{tabular}{|l|l|l|r|}
\hline & & Silicon & Steel \\
\hline Young's Modulus & $\mathrm{E}(\mathrm{Pa})$ & $1.70 \mathrm{E}+112.03 \mathrm{E}+11$ \\
\hline Torsional Modulus & $\mathrm{G}(\mathrm{Pa})$ & $5.10 \mathrm{E}+107.85 \mathrm{E}+10$ \\
\hline Density & $\rho\left(\mathrm{kg} / \mathrm{m}^{\wedge} 3\right)$ & 2330 & 7800 \\
\hline Yield Stress & $\mathrm{Sy}(\mathrm{Pa})$ & $6.90 \mathrm{E}+088.00 \mathrm{E}+08$ \\
\hline Torsion frequency coef. (maximize) & $(\mathrm{G} / \mathrm{\rho})^{\wedge} 0.5$ & 4678.505 & 3173.024 \\
\hline Other mode frequency coef. (maximize $)$ & $(\mathrm{E} / \rho)^{\wedge} 0.5$ & 8541.743 & 5106.499 \\
\hline Deformation coef. (minimize) & $\rho / \mathrm{E}$ & $1.37 \mathrm{E}-08$ & $3.83 \mathrm{E}-08$ \\
\hline Flexure stress coef. (maximize) & Sy/G & $1.35 \mathrm{E}-02$ & $1.02 \mathrm{E}-02$ \\
\hline
\end{tabular}

\section{SUMMARY REMARKS}

Different mechanical tradeoffs imposed by mode frequencies, dynamic mirror deformation, and flexure stress can be analyzed together using the analytical formulas given above. Optimal mirror and flexure dimensions that meet the resolution and frequency requirements of high-performance displays can be calculated. Even though analytical formulas have several simplifying approximations, they are useful for design tradeoff analysis. Final designs should be evaluated using FEA.

The scanner resolution figure of merit, $\theta D$, of Steel-based MRS and Silicon MEMS scanner are about 18deg.mm and 9deg.mm, respectively. As discussed above, Silicon has better mechanical properties than Steel and allows for higher frequency and resolution. We can conclude that the potential of MEMS is not yet fully realized. Higher performance levels can be achieved with MEMS scanners when all the engineering design tradeoffs and the fabrication related issues are better understood and resolved.

I'd like to thank Prof. Metin Muradoglu at KOÇ University and Frank DeWitt at Microvision Inc. for helpful discussions.

\section{REFERENCES}

[1] M. Bayer, "Retinal Scanning Display - a Novel HMD Approach to Army Aviation" Head and Helmet-Mounted Displays VII, Proc. SPIE Vol. 4711, Orlando, Florida, April 2002.

[2] H. Urey, Retinal Scanning Displays, to be published in Encyc. of Optical Engineering, Marcel-Dekker, 2002.

[3] H. Urey, N. Nestorovic, B. Ng, A. Gross, "Optics Designs and System MTF for Laser Scanning Displays," Helmet and Head-Mounted Display IV, Proc. SPIE Vol. 3689, p. 238-248, Orlando, Florida, March 1999.

[4] H. Urey, D. Wine, and T. Osborn, "Optical Performance Requirements for MEMS-scanner Based Microdisplays," Conf. on MOEMS and Miniaturized Sys., SPIE Vol. 4178, p.176-185, Santa Clara, CA, 2000. 
[5] D. W. Wine, M. P. Helsel, L. Jenkins, H. Urey, T. D. Osborn, "Performance of a Biaxial MEMS-Based Scanner for Microdisplay Applications," Conf. on MOEMS and Miniaturized Systems, SPIE Vol. 4178, p. 186-196, Santa Clara, California, 2000.

[6] J. S. Kollin, M. Tidwell, "Optical engineering challenges of the Virtual Retinal Display," Novel Optical Systems Design and Optimization, Proc. SPIE, Vol.2537, p.48-60, San Jose, California, 1995.

[7] J. Lee, Y. Mun, S. Do. Y. Ko, B. Choi, J. Kim, C. Hong, D. Jeon "Laser TV for Home Theater" Projection Displays VIII, Proc. SPIE Vol. 4657 San Jose, California, 2002.

[8] H. Urey, F. DeWitt IV, S. Luanava, "Optical scanners for high-resolution RSD systems," Head and Helmet Mounted Displays VI, Proc. SPIE, Vol. 4711, Orlando, Florida, 2002.

[9] G. Marshall, Ed., Optical Scanning, Marcel Dekker, New York, 1991.

[10] L. Beiser, R.B. Johnson, "Scanners," Ch. 19 in Handbook of Optics, 2nd ed., Part II, McGraw-Hill, NY, 1995.

[11] H. Urey, F. DeWitt IV, S. Luanava, "Optical scanners for high-resolution RSD systems," Head and Helmet Mounted Displays VI, Proc. SPIE, Vol. 4711, Orlando, Florida, 2002.

[12] H. Schenk, P.Dürr, Th. Haase, D. Kunze, U. Sobe, H. Lakner, H. Kück, "Large Deflection Micromechanical Scanning Mirrors for Linear Scans and Pattern Generation”, J. Selected Topics in Quantum Electronics, Vol. 6, No. 5, p.715-722, 2000.

[13] R. Conant, P.Hagelin, U. Krishnamoorthy, O. Solgaard, K. Lau, R. Muller, “A Raster-Scanning Full-Motion Video Display Using Polysilicon Micromachined Mirrors”, IEEE Transducers'99, Sendai Japan, p. 376-379, 1999.

[14] H. Schenk, P.Durr, D Kunze, H. Lakner, H. Kuck, "An Electrostatically Excited 2D-Micro-Scanning-Mirror with an In-Plane Configuration of the Driving Electrodes", IEEE MEMS Proceedings, Vol. 13, p. 473-478, 2000.

[15]D. Dickensheets, G. KiNo, "Microfabricated biaxial electrostatic torsional scanning mirror", Proc. SPIE, Vol. 3009, p.141-150, 1997.

[16] U. Hofmann, S. Muehlmann, M. Witt, K. Dorschel, R. Schutz, B. Wagner, "Electrostatically Driven Micromirrors for a Miniaturized confocal Laser Scanning Microscope", Miniaturized Systems with MicroOptics and MEMS, Proc. SPIE, Vol 3878, p. 29-38, 1999.

[17] L Fan and M. Wu, "Two-Dimensional Optical Scanner with Large Angular Rotation Realized by SelfAssembled Micro-elevator", Broadband Optical Networks and Technologies: An Emerging Reality, IEEE LEOS Summer Topical Meetings, p. 107-108, 1998.

[18] H. Lakner, W. Doleshal, P.Durr, A. Gehner, H. Schenk, A. Wolter, G. Zimmer, "Micromirrors for Direct Writing Systems and Scanners", Miniaturized Systems with Micro-Optics and MEMS, Proc. SPIE, Vol. 3878, p. 217-227, 1999.

[19] S. Schweizer, P.Cousseau, G. Lammel, S. Calmes, Ph. Renaud, "Two-dimensional thermally actuated optical microprojector" Sensors and Actuators, Vol. 85, p. 424-429, 2000.

[20]P. M. Hagelin and O. Solgaard, "Optical Raster-Scanning Displays Based on Surface-Micromachined Polysilicon Mirrors", IEEE Journal of Selected Topics in Quantum Electronics, Vol. 5. No. 1, 1999.

[21]H. Schenk, "Ein neuartiger Mikroaktor zur ein- und zweidimensionalen Ablenkung von Licht", Ph.D Thesis, p. 92, Gerhard-Mercator-Universität Duisburg, (http://www.ub.uni-duisburg.de/diss/diss0101/), 2000.

[22] H. Miyajima, N. Asaoka, M. Arima, Y. Minamoto, K. Murakami, K. Tokuda, K. Matsumoto “An Electromagnetic Optical Scanner With Polyamide-Based Hinges" Proc. IEEE Transducers'99, Sendai, Japan, 1999.

[23] M. Liang, "Performance characterization of a single bi-axial scanning MEMS mirror based head-worn display", Proc. SPIE, Vol. 4773, Seattle, WA, July 2002.

[24] W. C. Young, Roark's Formulas for Stress and Strain, 6th ed., pg. 348, McGraw-Hill, 1989.

[25]P. J. Brosens, "Dynamic mirror distortions in optical scanning," Applied Optics, vol. 11, p. 2988-2989, 1972. 